\title{
Adaptive Time Difference of Time of Arrival in Wireless Sensor Network Routing for Enhancing Quality of Service
}

\author{
Vignesh Prasanna Natarajan*, Kavitha Thandapani \\ Department of Electronics and Communication Engineering, Vel Tech Rangarajan Dr.Sagunthula R\&D Institute of Science \\ and Technology, Chennai 600062, India
}

Corresponding Author Email: vigneshprasanna@veltech.edu.in

https://doi.org/10.18280/i2m.200602

Received: 18 September 2021

Accepted: 3 December 2021

\section{Keywords:}

underwater wireless sensor networks, energy efficiency, convex depth variance routing, network lifetime, adaptive time difference

\begin{abstract}
Underwater wireless communications are critical in military and corporate operations such as environmental monitoring, underwater exploration, and scientific data collection. Existing protocols for terrestrial wireless sensor networks (TWSNs) perform poorly in terms of energy efficiency, dependability, and transmission. Because they have separate qualities, they cannot be used directly in the UWSN. The present challenges include developing an EDVR algorithm for determining the distance to each node and the variance in node depth in order to estimate energy consumption reductions. This technique takes the depth of the two-hop neighbors into account and calculates the time aid from the Adaptive Time Difference of Arrival (ATDoA), which is avoided by broadcasting information to its neighboring node, with farther forward nodes. To determine the time difference between the reception of two signals at a node, the adaptive time Difference of time of arrival (ATDoA) is easier to measure than the time at which the signal arrives. In the UWSN, the following transmission assigns higher node energy if the node is lower. It increases system performance, saves lives, and minimizes packet wait time at the destination. The results show that nodes have a longer lifetime, fewer dead nodes, use less energy, and take less time to propagate than techniques.
\end{abstract}

\section{INTRODUCTION}

The Underwater Wireless Sensor Networks (WSN) monitor consists of a number of sensor nodes that are placed according to a given cube in the water. The UWSNs overall monitoring area must be able to be constructed for each sensor node to reach the sink plane of multi-hop path coverage, with a grounded sensor network to provide communication coverage to be covered by the sensor node. Data collection and environmental monitoring are clearly major players, as more research is being done on underwater systems. This raises the need for better methods of collecting data and screens environment. In recent years, we have also seen the rapid growth of sensor network applications in the underwater environment, and the establishment of Underwater Wireless Sensor Networks (UWSNs). Wireless networks are a technology that makes underwater applications possible. The underwater sensor network contains a variable number of vehicles and sensors placed in a given area to perform joint surveillance tasks.

The underwater sensor node communication between base station is represent in Figure 1. In underwater, several sensors are included to gather or transmit the data from different locations in under water and these communications are done with the use of the base station established. Due to the physical properties of the underwater diffusion media, the immersion of the sound is a sign of extreme broadcast unhappiness, many perspectives are subject to low propagation speed time, isolation and Doppler elongation, and is badly affected by high transmission delay. There are various factors that influence
UWSNs communications: Energy Consumption, Transmission Loss and High Bit Error Rates.

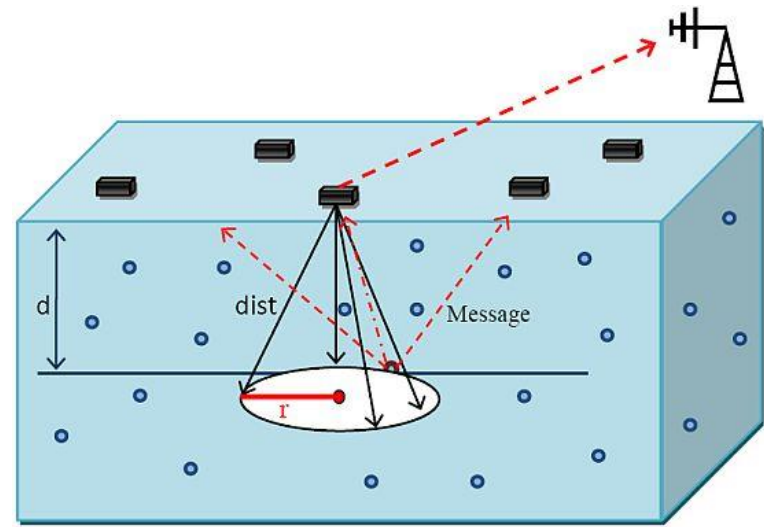

Figure 1. Underwater sensor network communication

Energy Consumption: The battery power runs underwater sensor node. With UWSNs, it is usually not possible to replace or recover disconnected or drained batteries at sensor points. A small number for these basic purposes, and the main purpose is to extend battery life isolation and other performance. The power of the sensor terminal is very important for efficient radio transmission and operation. These are the source of energy controls that are generally unmanned.

High Bit Error Rates: UWSNs suffer from severe multiway fading. The multi-way effect in the aquatic environment is due to the reflection from the wave reflection on the water 
below and for other reasons, and the rate of sound change. The signal strength received is significantly reduced by the multiway waves that are the fading of the relay.

Transmission Loss: Transmission loss in the underwater environment is due to geometric diffusion loss and abrasion. The geometric scattering loss phenomenon is due to the propagation of sound signals. Depletion is done by the sound energy story. The term geometric diffusion can define wave power as propagation loss to save energy. The wave energy decreases as it propagates over long distances from the wave source and fills a large area of the signal. Resistance to abrasion underwater media may define a reduction in signal or signal loss. As the signal travels through the underwater medium, the signal strength decreases with distance. As a result, underwater contact is obtained.

Energy Consumption: The energy consumption in wireless sensor networks represents the usage of allocated energy levels by the nodes in sensor network to perform the data communication operations. The energy consumption is to be continuously monitored for performance enhancement of the network.

High Bit Error Rate: The bit error ratio (also known as BER) is the quantity of bit errors divided by total number of transmitted bits during the time frame under consideration. Bit error ratio is a dimensionless quantity performance metric that is frequently stated as a percentage. This estimate is correct for a wide time range and a large number of bit errors.

Sensors have fixed transmission power, small memory to store the sensed data, transmission unit to transit the data and receiving unit to receive data. These nodes operate on the battery. Recharging of this battery is just not possible because of higher cost. Simple nodes are usually set at the bottom of the ocean or attached to the seabed through some cord. These sensor nodes can communicate to the other nodes or sink through the various signals available for communication.

\section{RELATED WORK}

Underwater Wireless Sensor Networks (UWSNs) has many challenges, but one of the main challenges is limited battery power. This scenario is based on the fact that in some cases the node positions and positions are replaced by frequent water currents and the presence of further deterioration to the cluster head or other nodes. For the above reasons, it should be adaptable to changes in energy efficiency and network routing to UWSN [1]. Application of Wireless Sensor Network (WSN) to various problems in posture in an underwater environment. One of the most important issues is the limited life of the underwater sensor terminal [2]. The energy harvesting method is presented as a promising solution, which challenges by changing the battery of the sensor node in an underwater environment and considers an expensive method. If a node wants to send the data package, it sends the spreadsheets and other packets for a multi-way delay and input. Protocol High network performance, low transmission delay and low power consumption can be achieved compared to traditional protocols [3] of UWANs (Underwater Acoustic Networks). The model when compared to the previous models exhibits better performance but the time complexity levels are high in this model that need to be overcomed.

The underwater acoustic WSN exhibit design has significant advantages in terms of sensor requirements, Bit Error Rate (BER), and power consumption in uncoordinated
COFDM communication systems. It also analyzes the sensor placement plan and finds areas where the proposed plan was able to reduce power consumption and improve BER [4]. Dummy source nodes and dummy data streams are commonly used in wireless sensor networks, so there is a need for a way to have similar functions in UASNs. Therefore, it matched the new pseudo-source node and added randomness to the very underwater masonry structure of the underwater network [5]. For this purpose, when sensors collect data, time and place are fundamental aspects, especially in the case of locational data. Many researchers are considering the location of underwater sensor nodes, taking into account the location of sensors where data was collected and mostly concentrated on fixed sensor nodes [6]. The communication process in this model is delayed because of the attacks om the sensors that are to be identified for improving the efficiency of the model.

Used in underwater research to predict underwater earthquakes, tsunamis, pollution, such as UWSN, but in terms of time delay, bandwidth, speed, and other low bandwidth and high localization, UWSN is land-based radio. It is different than a sensor network. UWSN Delay Operating Policy Ground WSN [7] Designed protocols are not suitable for use. To obtained various studies on marine resources such as underwater and disaster prevention information. However, the standards for security and authentication protocols used for underwater wireless sensor networks (UWSNs) are incomplete [8]. To combat the prevalent shadowing phenomenon of underwater communication channels, integrated WSNs employ parallel coding systems and networks [9] coding. A set of spatial domains and an LDPC coded OFDM system are presented as the coding approach [10].

In this context, water monitoring plays an important role in detecting water pollution. It participates in the public awareness of the local water resources, participates in the basic surveillance of local water bodies [11], and makes many efforts to really invest in building public awareness of the protection of the world's citizens. Did. In recent years, where wireless sensor network (WSN) security has received a lot of attention, malicious attacks have been caused by system vulnerabilities. The transmission capability of wireless media allows an intercept signal to easily intercept signals on a physical channel. To protect this private data [12] wireless sensor networks are an important issue for users to share as they are found among users. The water pollution detection levels can further be enhanced by considering the particles with more samples to improve the accuracy rate.

The efficiency of an underwater sensor network depends on the energy capacity of the operating nodes, and the number of network lives. Therefore, the number of active nodes plays an important role in the performance of the system. However, due to the number of working nodes in a harsh underwater environment, it changes frequently over time for various artificial and natural reasons (e.g., some nodes may be faulty, some may be damaged, or batteries May fail) [13]. In these two methods, data packets are forwarded in a multi-hop manner in the virtual pipe of the sensor node. Nodes outside the tube make forward data packets to prevent network flooding. Sharing toward the gaps in the network at each hop is avoided by using 2-hop information [14].

Military espionage research is an important field in applications ranging from target detection, motion assessment and accurate forecast security to daily life transport and medical application. View From a national security 
perspective, surveillance and safety programs should remain intact to prevent the threat of explosives, enemy submarines, and other diving vessels $[15,16]$ to ports, harbors, and waterways. It is the basis for forwarding messages and sensing data collection and transmission in routing, underwater radio sensor networks, and less expensive protocols that consume energy. However, most of the communication gap issues do not handle well, and when forwarding [17] messages, the quality of the link with the sensor node is not taken into account. As a result, for sensor deployment with the goal of decreasing energy consumption in a 3D underwater environment, a deterministic deployment technique is advocated. The purpose of this optimization issue for sensor placement is to optimise the least number of sensors [18] and target coverage.

Base Station PS is a wireless sensor network (wireless) that consumes energy to transmit data to each sensor, considering various research areas to temporarily store recorded data while knowing the circumstances. There is a problem related to this (sensor networks). This is because the data [19] is sent through multiple nodes before it can reach further processing PS. The integrated battery that operates the underwater sensor terminal uses the supplied power and replacing the underwater battery is a significant challenge, especially with the large number of sensor terminals. Energy efficiency here faces an important issue. In addition to energy efficiency, there is the fact that UWSN usually produces another basic theme for sensitive data [20] due to the confidentiality of the data.

Many routing algorithms for UWSN have been reviewed in this section. Some algorithms were having issue of fast energy depletion, shorter lifetime, network coverage, sparse density and void regions in network. Here problem of void regions has been taken and provided solution for avoiding void regions using residual energy and depth variance. A location free routing protocol named Energy Efficiency Convex Depth Variance Routing (ECDVR) has been proposed here to avoid the void regions.

\section{PROPOSED METHODOLOGY}

This proposed method to introduce an Energy Efficiency Convex Depth Variance Routing (ECDVR) to achieve the energy optimization and node transmission error problem. In this method using a Convex Position Estimation method to estimate the node position and location using a convex matrix. A concentric set is a set of points that can be connected to any two points in a set that include a path containing the points contained in the set. The path of the sensor network between the two terminals is a concentric moment of collection with all the nodes, creating only the nodes which interact with the set of sensor nodes. The Adaptive Time Difference of Arrival (ATDoA) method allows information to be used to estimate the difference in distance between two nodes. The time difference of arrival time is used to measure the fault tolerance and estimate the distance between these two nodes. In this Figure 2 represents the block diagram of the proposed method. The sink Head has a priority value it will do the Aggregation of all the data and path. Initially, all priorities are set to zero value. Sink receives values and finds in which priority this value falls and increases the value of that aggregated value.

In Figure 2, initially the network is established and then network clusters are formed that considers a group of nodes as a cluster and then forms a subnetwork and then the convex position estimation is calculated. The proposed ATDoA model is applied on the clusters generated and then the sink node selection is performed that is used for monitoring and analysis. The energy estimation of every node is calculated to check the energy consumption levels and the performance also gets impacted with energy consumptions. Using the routing table, with base station routing, the data among the sensors will be transmitted.

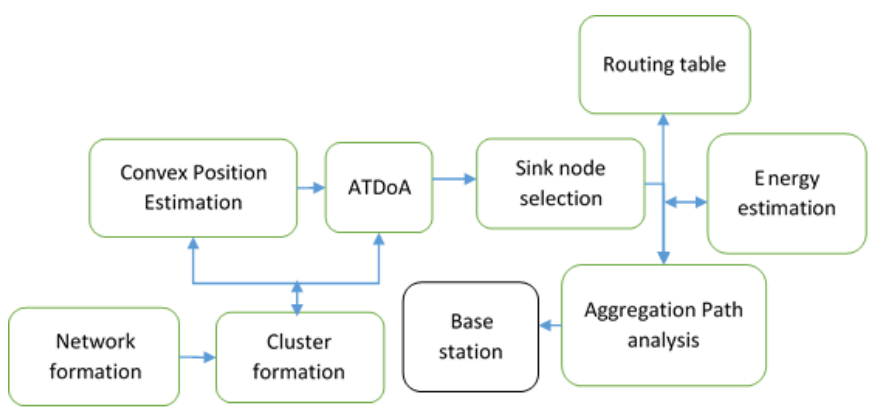

Figure 2. Proposed method block diagram

Special nodes are used to form clusters, which periodically form clusters by transmitting cluster request message. The sensor nodes who listen to that particular message are similarly arranged in their which forms a cluster. Transmitter Node The transmitter node converts the next node data packet with less depth to more energy. If the sender does not have multiple neighboring nodes at shallow depths, it will share a high energy node data packet. The following are considered for the UWSN network format. All sensor nodes can move freely in all directions. All the node information, node id is store in the sink node and every node initial energy is $E_{\text {init }}$. The transmission path satisfied the aggregate value it transmits the data to the base station.

\section{a. Convex position estimation}

When the ordinary node got the information from at sink nodes, they can start next step of algorithm to calculate their positions by using a Convex Position Estimation. In under water sensor networks, $\mathrm{z}$ coordinates of nodes are assumed to be calculated by the pressure sensors. By calculating the depth of the unknown node, Eq. (1).

$$
\begin{aligned}
\left(n X_{1}-n x_{2}\right)^{2}(i) & +\left(n Y_{1}-n y_{2}\right)^{2}(i) \\
& +\left(n Z_{1}-n z_{2}\right)^{2}(i)=D^{2}
\end{aligned}
$$

In this Proposed location estimation method is better than Lateration and Min-Max in terms of less complexity and low localization errors. Where $(\mathrm{nX}, \mathrm{nY}, \mathrm{nZ})$ are the estimated location coordinates of the unknown sensor node $\mathrm{Ni}$, (nx, ny, $\mathrm{nz}$ ) are the coordinates of i's location of sink node, D is the distance in concerning the unknown node $\mathrm{Ni}$ and the sink node i.

\section{Algorithm steps}

Step 1: The network topology to be construct and node are deployed randomly on the coordinate point $\mathrm{x}, \mathrm{y}, \mathrm{z}$.

Step 2: Distance Estimation is done in this step by sending a message to other node (n).

For (each node $\mathrm{n}$ to $\mathrm{i}$ )

$n_{i}$ send signal to $n_{i+1}$ at location $l_{1}$

$n_{i+1}$ receives this location $l_{2}$. 
End for

For (each node $\mathrm{n}$ to $\mathrm{i}$ )

End for

$n_{i+1}$ send a replay message its id at $l_{3}$

Consider

Step 3: Repeatedly Select sink Node for all unknown nodes.

Step 4: Calculate Location of node using the Eq. (1).

Step 5: Calculate Localization error $(\boldsymbol{\rho})$ as per Eq. (2).

$$
\begin{gathered}
\rho=\sum_{i}^{n}\left(\left(n X_{1}-n x_{2}\right)^{2}(i)+\left(n Y_{1}-n y_{2}\right)^{2}(i)+\right. \\
\left.\left(n Z_{1}-n z_{2}\right)^{2}(i)-d(i)\right)
\end{gathered}
$$

After the location estimation localization error is calculated if the error is less than 1 then its location is confirmed and this $\mathrm{N} 1$ can be act as anchor node for other nodes and broadcast its location to other nodes.

\section{b. Cluster formation}

Once the nodes are deployed, the base sink sends out HELLO packets and determines the position of advanced nodes and creates a concentric cluster around them. To select another cluster head node using selection algorithm. These cluster heads thereby form a cluster and broadcast advertising messages to all its neighbors.

\section{Algorithm}

Step 1: Residual energy $E_{\text {init }}$ at each node.

Step 2: Balanced clusters and CHs.

Step 3: compute the average residual energy of all nodes $A_{e}=$ $\sqrt{2 \varepsilon_{\text {init }}-T_{r e}}, / / T_{r e}$-Total residual energy.

Step 4: The remaining energy of the group of selected nonsensing nodes (s) is higher than the average level.

Step 5: Select the two nodes with the maximum separation distance between them in the cluster head $\mathrm{S}$.

Step 6: Specify all other nodes with their closest cluster head, leading to the formation of two large groups.

Step 7: Element Divided into two groups S1 and S2 that are members of two groups $\mathrm{S}$.

Step 8: Until N cluster is selected.

This allows several nodes to share the same high threshold energy with the same frequency channel by splitting the signal into different time slots. The cluster head aggregates the data from all the nodes in the cluster and this aggregated data is transmitted to the advanced node which in turn re-routes the information to the sink node.

\section{c. Adaptive time difference of arrival}

The bit error ratio is the number of bit errors divided by the total number of transmitted bits for the time period in question. The bit error ratio (BER) is a dimensionless quantitative performance metric that is commonly expressed as a percentage. This estimate is accurate over a wide time interval and with a big number of bit mistakes.

\section{Algorithm step:}

Step 1: The Sink Node(SN) monitors the movement of $Q$ in a sequential pattern.

Step 2: Then the SN analyzes the movement pattern as O[SN, $\mathrm{CN}, \mathrm{DN}]$.

Step 3: SN Estimate the Transmission support (Sup[O]) every node

$$
\operatorname{Sup}[\mathrm{O}]=\{L \in D N \mid O \in O[L]\} \quad / / \mathrm{L} \quad \text { List }
$$

containing node pair detected with time, $\mathrm{O}$ Transmission

$$
\mathrm{L}=\left\{p n_{1}, t_{1}, p n_{2}, t_{2}, p n_{3}, t_{3}, \ldots \ldots p n_{m}, t_{m}\right\} s_{i} \in s / /
$$

partipation node $\mathrm{pn}$ and time $\mathrm{t}$

Step 4: SN Estimate the Conformation Transmission (Con[O]) $\operatorname{Conf}(\mathrm{O})=[\mathrm{SN}, \mathrm{CN}] \rightarrow[\mathrm{DN}]$ $=\operatorname{Sup}([\mathrm{SN}, \mathrm{CN}, \mathrm{DN}]) / \mathrm{Sup}([\mathrm{SN} . \mathrm{CN}])$

Step 5: SN measure Receiving signal strength $R S S_{p n}$ from $\mathrm{O}[\mathrm{SN}, \mathrm{CN}, \mathrm{DN}]$.

$$
\text { If } R S S_{p n}>R S S_{t h} \quad \text { //Threshold value }
$$

End if Add to Routing list $\mathrm{L} \leftarrow\left[S N_{i d}, C N_{i d}, D N_{i d}\right]$

If $\mathrm{O}$ is repeating many times, then

If $\mathrm{T}$ traverse the near to SS and CS and Conf $[\mathrm{O}]$ is high, then

Next SN to be traversed is DS End if

End if

These methods can use multiple signals. If the sound signals have different speeds and are transmitted simultaneously by the transmitter at the known time, the time $\mathrm{T} 1$ and $\mathrm{T} 2$ by the receiver will be received for their speed variations respectively.

\section{d. Aggregation path analysis}

At first, it determines the total value of the sensed node information. Following that, it works on run duration and generates a string, storing the value for previously sensed information. This approach minimises the size of the generated packet and ensures that the values delivered are time synchronised. Aggregation Path analysis is based on the number of data processes and data drops at each node. When an aggregate request is received by a sink node, it generates a packet containing data in bit form. The string generated by the sink node is transformed into bits after it has been concatenated with the current sensed value and its aggregated value. If the sensed value is critical, the sensor will raise the priority of the packet corresponding to this value and promptly deliver it to the sink head. Otherwise, it will only transmit when the aggregation request is received.

\section{Algorithm steps:}

Step 1: Forward the packet to forwarder neighbor.

Step 2: Initialize packet queue as Queue.

Step 3: Initialize receiver node set as Ri.

Step 4: NFi is the next forwarder node list.

Step 5: Si sense the environment. Create a sensed data packet. Step 6: If $(n>80)$

Generate packet;

Priority $=2$;

Send packet towards sink Head;

Step 7: Else if $(n>50 \& \& n<=80)$

Generate packet;

Priority $=1$;

Else

Send packet towards sink Head;

End if

$$
\text { Collect data in Queue. }
$$

If the sensed value is greater than 80 then sensor will generate a packet and immediately send it towards Cluster Head which further sends this packet to sink without waiting. Sensor nodes sense data and send this data at periodic time interval. If sensed value is less than 50 then there is no need to send packet and sensor does only sensing and storing tasks. The network adjustment is used to pull back the node to its last location when it was in range of some cluster head. In which case the nodes have to go to its last stored place in the gold 
memory where it is to be tracked.

\section{RESULT AND DISCUSSION}

The simulation developed in the NS-2 tool with OTCL (Object Tool Command Language) it's similar to objectoriented language. In these tools used for network QoS parameter analysis like throughput, delivery ratio, time delay are evaluated. This section presents a relative analysis of the results obtained therefrom.

Table 1. Simulation parameters

\begin{tabular}{cc}
\hline Parameters & Values \\
\hline Number of nodes & 200 \\
Data size & $150 \mathrm{Mb}$ \\
Packet size & $512 \mathrm{~Kb}$ \\
Transmission protocol & $\mathrm{TCP}$ \\
Payload Size & $576 \mathrm{bits}$ \\
Simulation time & $10 \mathrm{~min}$ \\
Starting energy & $10 \mathrm{~J}$ \\
\hline
\end{tabular}

In this above Table 1 is show the proposed method developed simulation parameter to analysis the UWSN performance. in this proposed method Energy Efficiency Convex Depth Variance Routing (ECDVR) compare to existing method distance-vector-based opportunistic routing (DVOR), depth-based routing (DBR) and DUOR methods.

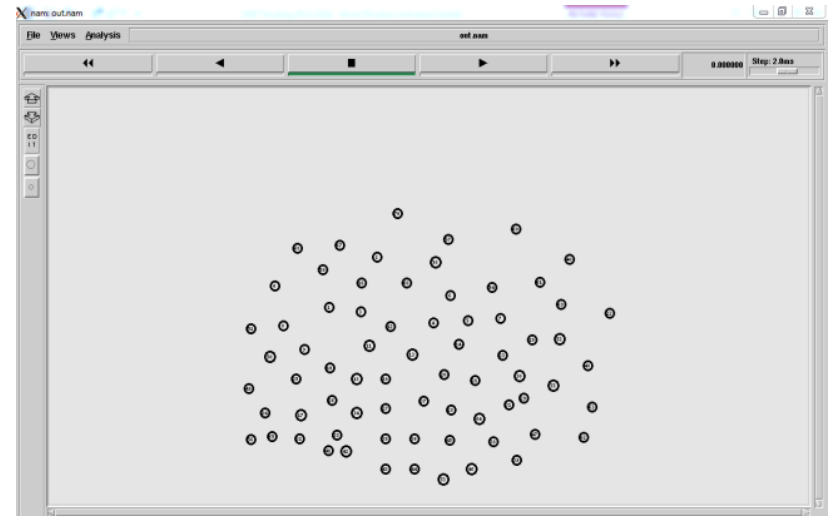

Figure 3. Process of node deployment

In this process of node deployment is show in Figure 3. The nodes are randomly formation on $\mathrm{X}$ - axis and $\mathrm{Y}$-axis in Network Animetor (nam) window.

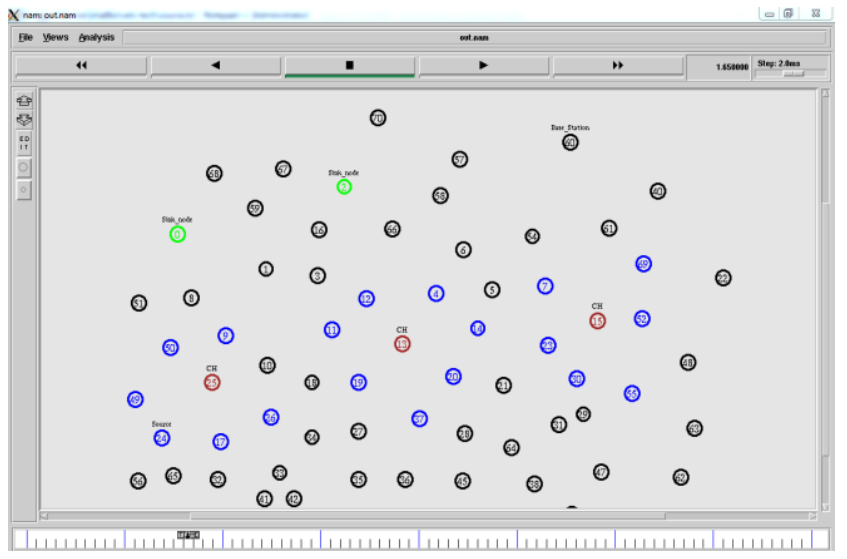

Figure 4. Cluster formation and sink node selection

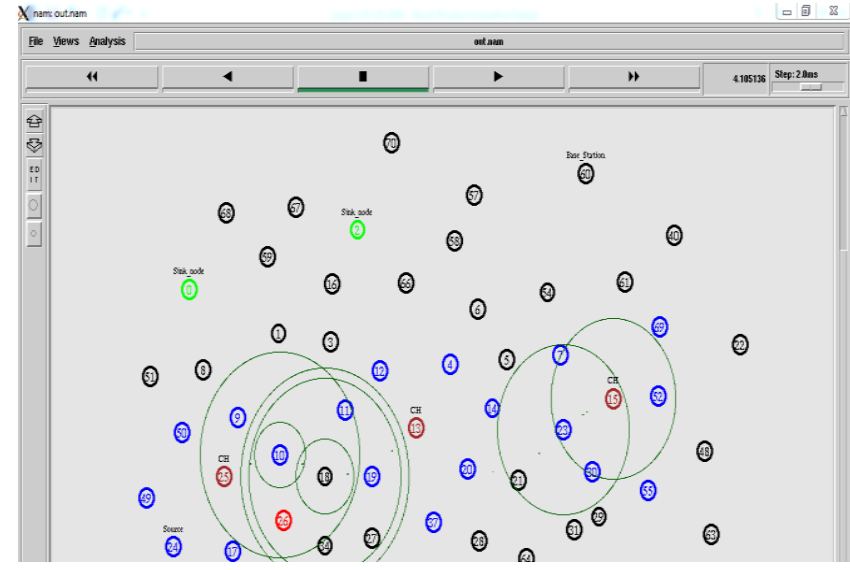

Figure 5. Dynamic cluster change high energy node

The cluster head broadcasts the message to the other node after node deployment to select cluster head for high power node. Figure 4 depicts the process of cluster creation and sink node selection in which someone will add its clusters in response to the other end. The Figure 5 represents the dynamic cluster change energy node.

The throughput ratio to analyze the number of packets send divided by the total time to taken the Eq. (3) follow.

$$
\text { throughput ratio }=\frac{\text { packet size } * \text { send packet } * 8}{1000}
$$

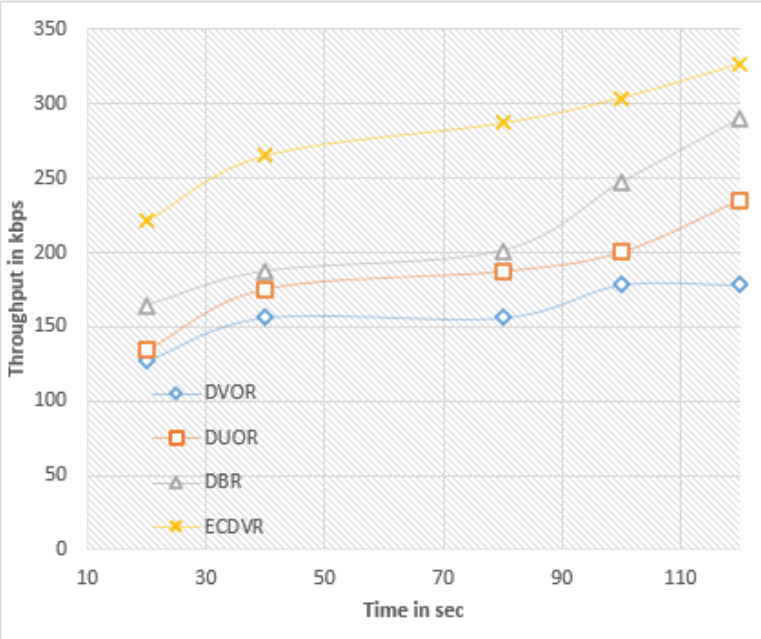

Figure 6. Comparison of throughput ratio

Figure 6 represents an existing method and proposed method throughput ratio. In this analysis of existing methods have lower throughput values than ECDVR because the data is sent over an unrecognized relay gap base station, especially at the border. The throughput of the ATDoA system is improved by increasing the number of nodes.

At this E2E delay (end to end) time analysis, the proposed method packet transmission time is taken, and receiving time divided by the number of connections in UWSN.

$$
\begin{aligned}
\text { End to end Delay } & =\text { Arrival time } \\
& - \text { Sent time } \\
& / \text { Total number of connections }
\end{aligned}
$$

Table 2 is the proposed approach and provides an end-toend delay comparison obtained through the two existing approaches. 
Table 2. Analysis of end to end delay

\begin{tabular}{ccccc}
\hline $\begin{array}{c}\text { Number of } \\
\text { node }\end{array}$ & $\begin{array}{c}\text { DVOR in } \\
\text { sec }\end{array}$ & $\begin{array}{c}\text { DUOR in } \\
\text { sec }\end{array}$ & $\begin{array}{c}\text { DBR in } \\
\text { sec }\end{array}$ & $\begin{array}{c}\text { ECDVR in } \\
\text { sec }\end{array}$ \\
\hline 50 & 1.2 & 2 & 2.4 & 1 \\
100 & 0.91 & 1.5 & 2 & 0.7 \\
200 & 0.8 & 1.42 & 1.9 & 0.5 \\
300 & 0.7 & 1 & 1.74 & 0.5 \\
500 & 0.6 & 0.95 & 1.2 & 0.3 \\
\hline
\end{tabular}

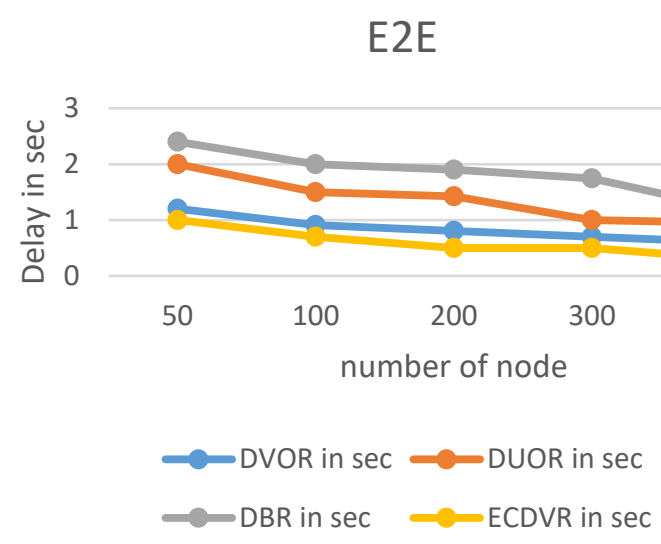

Figure 7. End to End delay

Above Figure 7 represents the end to end delay comparison of existing and proposed method. To this conclusion the proposed ECDVR method end delay is compared to other methods DBR, DUOR and DVOR which have a short time delay at performance.

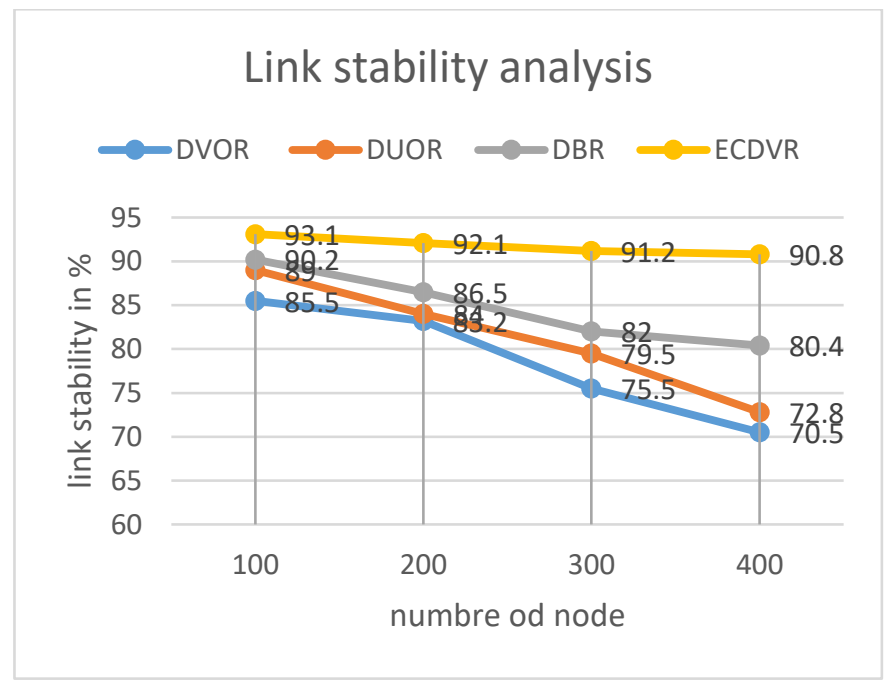

Figure 8. Link stability analysis

Figure 8 shows the link stability between base station and sink node performance of proposed ECDVR and the existing DBR, DUOR and DVOR method. The analysis is taken the number of nodes in this proposed method ECDVR has $90.8 \%$ for 400 nodes. It provides higher stability compared to the existing method DBR, DUOR and DVOR.

In each iteration of ECDVR, the energy used is estimated by the continuous use of energy within the node and network.

Figure 9 provides the comparison of the energy efficiency obtained by the proposed approach and the two existing approaches. At this analysis of energy consumption performance of the proposed ECDVR method, less time delay compared to other existing method DBR, DUOR and DVOR.

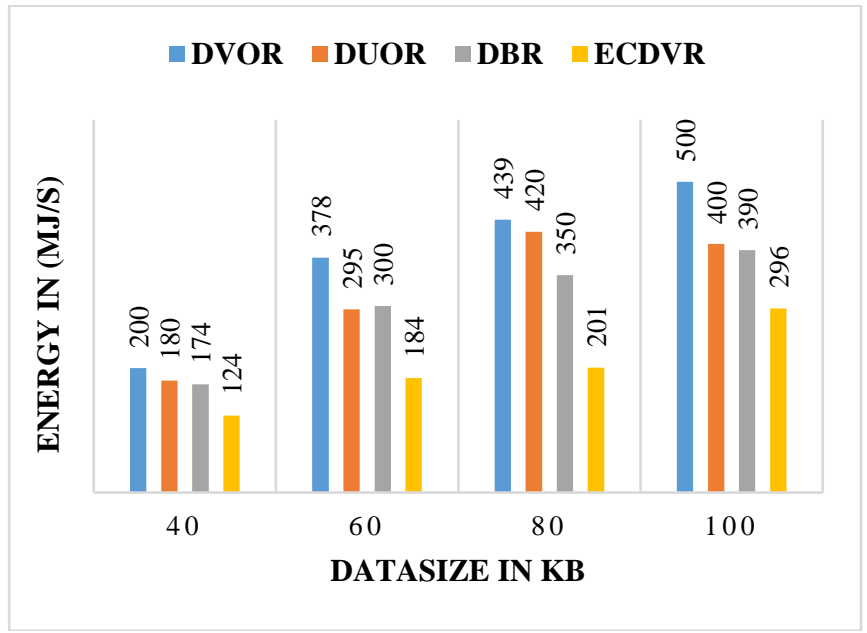

Figure 9. Analysis of energy consumption

The packet delivery ratio to analysis number of packet received divided by number of packet send.

$$
\begin{aligned}
& \text { packet delivery ratio } \\
& =\frac{\text { number of the packet received }}{\text { number of packet send }}
\end{aligned}
$$

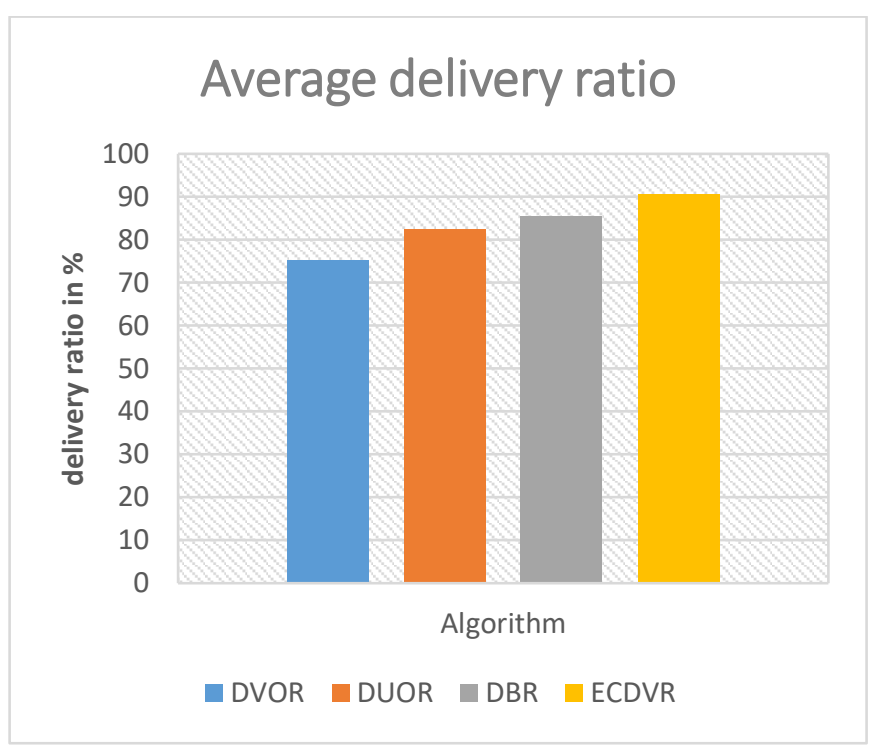

Figure 10. Analysis of Average packet delivery ratio

Figure 10 shows the average packet delivery ratio of existing and proposed method ECDVR. In this result, the proposed method ECDVR is $90.5 \%$ average packet delivery performance; similarly, the existing method DBR, DUOR provide an $84.3 \%, 75 \%$ data delivery ratio.

\section{CONCLUSION}

In this work, to improve the lifetime of UWSN without affecting the quality of the network. This work focuses on designing efficient routing protocols which would be beneficial for improving the lifetime of the network. Convex Position Estimation and Convex Position Estimation be used to localize the location of the unknown nodes and monitor the 
position of the node to reduce the localization error. Adaptive Time Difference of Arrival (ATDOA) is tracking the source and destination node transmission time each hop to achieve the short time transmission. In this proposed Energy Efficiency Convex Depth Variance Routing (ECDVR) algorithm have improved network lifetime, lesser number of dead nodes, lesser energy consumption, and lesser propagation delay. Network deployment technique for effective communication by maximizing coverage and larger network connectivity, higher network lifetime. This proposed method has better result, $327 \mathrm{kbps}$ of throughput ratio, 90.8\% link stability between two hop, $296 \mathrm{mj}$ energy consumption with $0.3 \mathrm{sec}$ delay compare to existing method.

\section{REFERENCES}

[1] Sofi, S.A., Mir, R.N. (2017). Natural algorithm based adaptive architecture for underwater wireless sensor networks. 2017 International Conference on Wireless Communications, Signal Processing, and Networking (WiSPNET), Chennai, India. https://doi.org/10.1109/WiSPNET.2017.8300179

[2] Erdem, H.E., Gungor, V.C. (2017). Lifetime analysis of energy harvesting underwater wireless sensor nodes. 2017 25th Signal Processing and Communications Applications Conference (SIU), Antalya, Turkey. https://doi.org/10.1109/siu.2017.7960419

[3] Fan, G., Chen, N., Wang, X. (2016). A cross-layer contention-based MAC protocol for underwater acoustic networks. 2016 3rd International Conference on Systems and Informatics (ICSAI), Shanghai, China. https://doi.org/10.1109/icsai.2016.7811034

[4] Iqbal, Z., Lee, H.N. (2016). Spatially concatenated channel-network code for underwater wireless sensor networks. IEEE Transactions on Communications, 64(9): 3901-3914.

https://doi.org/10.1109/tcomm.2016.2593746

[5] Han, G.J., Wang, H., Ansere, J.A., Jiang, J., Peng, Y. (2020). SSLP: A stratification-based source location privacy scheme in underwater acoustic sensor networks. IEEE Network, 34(4): 188-195. https://doi.org/10.1109/mnet.001.1900478

[6] Ullah, I., Liu, Y.M., Su, X., Kim, P. (2019). Efficient and Accurate Target Localization in Underwater Environment. IEEE Access, 7: 101415-101426. https://doi.org/10.1109/access.2019.2930735

[7] Radha, S., Bala, G.J. (2017). Flooding in static and free deployment for underwater wireless sensor networks. 2017 International Conference on Signal Processing and Communication (ICSPC), Coimbatore, India. https://doi.org/10.1109/cspc.2017.8305870

[8] Yun, C.W., Lee, J.H., Yi, O., Park, S.H. (2016). Ticketbased authentication protocol for Underwater Wireless Sensor Network. 2016 Eighth International Conference on Ubiquitous and Future Networks (ICUFN). https://doi.org/10.1109/icufn.2016.7537019

[9] Ilyas, N., Javaid, N., Iqbal, Z., Imran, M., Khan, Z.A., Qasim, U., Shoaib, M. (2015). AAEERP: Advanced AUV-aided energy efficient routing protocol for underwater WSNs. 2015 IEEE 29th International Conference on Advanced Information Networking and Applications. https://doi.org/10.1109/AINA.2015.169
[10] Iqbal, Z., Lee, H.N. (2015). Deployment strategy analysis for underwater cooperative wireless sensor networks. 2015 International Conference on Information and Communication Technology Convergence (ICTC). https://doi.org/10.1109/ictc.2015.7354642

[11] Khalfallah, Z., Fajjariz, I., Aitsaadiz, N., Langar, R., Pujolle, G. (2015). 2D-UBDA: A novel 2-Dimensional underwater WSN barrier deployment algorithm. 2015 IFIP Networking Conference (IFIP Networking). https://doi.org/10.1109/ifipnetworking.2015.7145293

[12] Huang, Y., Zhou, S., Shi, Z., Lai, L. (2016). Channel frequency response-based secret key generation in underwater acoustic systems. IEEE Transactions on Wireless Communications, 15(9): 5875-5888. https://doi.org/10.1109/twc.2016.2572106

[13] Sayem, A.S.M., Anower, M.S. (2015). A crosscorrelation based node estimation technique in an underwater wireless communication network. 2015 International Conference on Electrical \& Electronic Engineering https://doi.org/10.1109/CEEE.2015.7428254

(ICEEE).

[14] Javaid, N., Hafeez, T., Wadud, Z., Alrajeh, N., Alabed, M.S., Guizani, N. (2017). Establishing a cooperationbased and void node avoiding energy-efficient underwater WSN for a cloud. IEEE Access, 5: 1158211593. https://doi.org/10.1109/access.2017.2707531

[15] Joshy, A.A., Manuel, E.M. (2018). A novel approach for attaining energy-accuracy trade-off in WSN based underwater target tracking. 2018 IEEE Recent Advances in Intelligent Computational Systems (RAICS). https://doi.org/10.1109/RAICS.2018.8635073

[16] Xu, T., Hu, Y., Zhang, B., Leus, G. (2016). RSS-based sensor localization in underwater acoustic sensor networks. 2016 IEEE International Conference on Acoustics, Speech, and Signal Processing (ICASSP). https://doi.org/10.1109/icassp.2016.7472409

[17] Yao, B., Zhou, Z., Shu, L., and Xing, R. (2015). An energy-efficient routing protocol for underwater WSNs. 2015 IEEE 12th Intl Conf on Ubiquitous Intelligence and Computing and 2015 IEEE 12th Intl Conf on Autonomic and Trusted Computing and 2015 IEEE 15th Intl Conf on Scalable Computing and Communications and Its Associated Workshops (UIC-ATC-ScalCom), Beijing, China. https://doi.org/10.1109/UIC-ATC-ScalComCBDCom-IoP.2015.134

[18] Arivudainambi, D., Balaji, S., Poorani, T.S. (2017). Sensor deployment for target coverage in underwater wireless sensor networks. 2017 International Conference on Performance Evaluation and Modeling in Wired and Wireless Networks (PEMWN). https://doi.org/10.23919/pemwn.2017.8308032b

[19] Ghaleb, M., Felemban, E., Subramaniam, S., Sheikh, A.A., Qaisar, S.B. (2017). A performance simulation tool for the analysis of data gathering in both terrestrial and underwater sensor networks. IEEE Access, 5: 4190-4208. https://doi.org/10.1109/access.2017.2684539

[20] Uyan, O.G., Gungor, V.C. (2019). Lifetime analysis of underwater wireless networks concerning privacy with energy harvesting and compressive sensing. 2019 27th Signal Processing and Communications Applications Conference (SIU). https://doi.org/10.1109/siu.2019.8806241 